\title{
GREEN PEA SPROUT RESPONSE TO MICROBIAL INOCULATION AND INCREASING ATMOSPHERIC $\mathrm{CO}_{2}$ CONCENTRATION
}

Nahed H. Eissa ${ }^{{ }^{\star}}$, Mona S. Zayed ${ }^{2 \star}$, Hassanein ${ }^{1}$ M.K. and Abdallah ${ }^{3}$ M.M.F.

1- Central Laboratory for Agric. Climate, ARC, Giza, Egypt

2- Agric. Microbiology Dept., Fac. of Agric., Ain Shams Univ., P.O. Box 68, Hadayek Shobra 11241, Cairo, Egypt

3- Horticulture Dept., Fac. of Agric., Ain Shams Univ., P.O. Box 68, Hadayek Shobra 11241, Cairo, Egypt

$$
\text { *Corresponding author: monaszayed@agr.asu.edu.eg }
$$

\section{ABSTRACT}

Baby pea (pisum sativum L.) shoots is a new ready to eat baby leaf vegetable sprouts in Egypt. The overall quality change of baby pea shoots is greatly affected by surrounding environmental conditions especially increased elevation of carbon dioxide concentration in the air. This work focus on the impacts of predicted climate changes conditions on the quality of baby pea shoots by using two carbon dioxide concentrations (600 and 800 ppm) compared with ambient air (control) in interaction with three microbial inoculants and their combinations, in semi-automated growth chambers using tray sprouting method. The obtained results showed the largest yield of pea sprouts per unit area in 800 ppm CO 2 concentration with increasing about $20 \%$ more than ambient air (control) followed by 600 ppm with increasing about $9.4 \%$ than ambient air. Also, it revealed that using $\mathrm{CO}_{2}$ at 800 ppm increased pea sprout crude protein content $37.8 \%$, lipid $46.9 \%$ and energy $19.5 \%$ per unit area when compared to ambient air. While pea sprout treated by $800 \mathrm{ppm} \mathrm{CO}_{2}$ and inoculated by combination of $A z$. chroococcum $+B$. megaterium $+P$ s. fluorescens recorded the highest significant shoot length in the second cut and highest significant chlorophyll in first and second being 13.25 $\mathrm{cm}, 57.3$ and $58.9 \mu \mathrm{g} \mathrm{Chl./cm} \mathrm{(SPAD)} \mathrm{and} \mathrm{the}$ highest significant protein, lipids, and ash content being $48.65,4.95,10.69 \%$ as well as the highest significant mineral values of $\mathrm{P}, \mathrm{Ca}, \mathrm{Mg}, \mathrm{Fe}$ and $\mathrm{Zn}$ being $0.545,3.535,0.620 \%$ and 61.3 ppm respectively. Current study suggests that high $\mathrm{CO}_{2}$ concentration in the presence of $A z$. chroococcum $+B$.
\end{abstract}

megaterium $+P$ s. fluorescens improve the yield and the quality of baby pea shoots.

\section{INTRODUCTION}

Today, research seems to be confirming that seed sprouts are the function food of the future, as was the food of the past. Therefore, the attention of experts dealing with the healthy nutrition turned more and more towards, the determination of the biological value of the nutritional sprouts (Penas et al 2008; Abdallah 2008 and Maŕton et al 2010) The consumption of green leafy vegetables is recommended due to their high content of vitamins, minerals, and antioxidant phytochemicals, as well as low content of fat and carbohydrate (Rico et al 2007). The producers of fresh-cut sprouts seek for adding new varieties of leafy vegetables that are ready-to-eat to attract more consumers (Martinezsánchez et al 2012).

Pea shoots were recently presented as a ready-to-eat baby-leaf vegetable and is recognized as a popular vegetable in some parts of Asia and Africa which also is gaining popularity in the United States and Europe (Miles and Sonde 2003 and Santos et al 2014).

Baby pea shoots is considered as a healthy, beneficial and highly nutritive new leaf vegetable sprout (Ibrahim Mona 2015 and Ahmed et al 2018).

Changes in earth's climate have been projected by the end of the $19^{\text {th }}$ century because some atmospheric "greenhouse" gases are increasing at which Carbone dioxide $\left(\mathrm{CO}_{2}\right)$ one of them, (IPCC 2001). The naturally $\mathrm{CO}_{2}$ concentration in ambient 
air is 400 parts per million (ppm). However, doubling ambient $\mathrm{CO}_{2}$ level (i.e 700 to $800 \mathrm{ppm}$ ) which is predicted to occurs due to climate changes could make a significant and visible difference in plant growth and yield because $\mathrm{CO}_{2}$ is utilized by plants for higher rates of photosynthesis during daytime (Ludwig and Asseng 2006; Süß et al 2015 and Poudel and Dunn 2017).

However, $\mathrm{C}_{3}$ Photosynthetic pathways plants as peas are more responsive to higher $\mathrm{CO}_{2}$ concentration than plants having $\mathrm{a}_{4}$ pathway. An increase in ambient $\mathrm{CO}_{2}$ to $800 \mathrm{ppm}$ can increase the yield of $\mathrm{C}_{3}$ plants up to $40 \%$ to $100 \%$ (Poudel and Dunn2017).

The main consideration for biological management of plant growth is to utilize microbial inoculants that play a dynamic role in sustaining agriculture by improving their growth performance in a safer way (Mcdaniel et al 2014). Plant response to microbial inoculants could be associated with more than one mechanism at which microorganisms were suggested to have more than one function in stimulating plant growth that results in more than one consequence (Cakmakci et al 2007), therefore, they have great capabilities to increase plant growth and yield under different conditions. These increments could be attributed to different mechanisms such as increasing nutrients uptake through solubilization and degradation of complicated compounds, nitrogen fixation which has special effect on the physiological processes of plants (Valentine et al 2010; Zayed Mona 2012) and stimulating plant growth either by production of plant growth promoting substances such as indole3-acetic acid, cytokinins and gibberellins which able to encourage progressive effects on the plant growth and development or modulating endogenous plant hormone levels (Gray 2004; Van Loon 2007; Ortíz-Castro et al 2008; and Ahemad and Khan 2011), in addition to improving plant immunity against diseases by producing different antibiotics (Atta et al 2012).

These research focus on the impact of predicted climate change conditions (increased $\mathrm{CO}_{2}$ concentration) and different microbial inoculants on the growth and yield of baby pea shoot (green sprouts). The main objectives are to evaluate the effect of increased $\mathrm{CO}_{2}$ concentration on photosynthetic pigment (SPAD reading), chemical composition (proximate analysis), energy and mineral contents of pea shoot in the presence of different microbial inoculants.

\section{MATERIALS AND METHODS}

This study was carried out during winter seasons of 2015 and 2016 at Central Laboratory for Agricultural Climate (CLAC), Agriculture Research Center (ARC), Ministry of Agriculture and Land Reclamation, in Semi-automated control environmental chambers. The experiment was designed to study the effect of different microbial inoculants and two $\mathrm{CO}_{2}$ concentrations on pea sprout characters, yield and chemical composition in the two cutting.

\section{Microbial inoculants}

Three different bacterial strains have various potential activities, namely Azotobacter chroococcum, Bacillus megaterium and Pseudomonas fluorescens, were used in this study. They were kindly provided by Microbial Inoculants Center, Fac. Agric., Ain Shams Univ., Cairo, Egypt. Each strain was maintained in its appropriate medium. Azotobacter chroococcum was maintained on Modified Ashby's medium (Abd El-Malek and Ishac 1968) for 7 days $/ 30^{\circ} \mathrm{C}$, Bacillus megaterium was maintained on nutrient broth medium (Jacobs and Gerstein 1960) for $24 \mathrm{~h} / 30{ }^{\circ} \mathrm{C}$ while Pseudomonas fluorescens was maintained on King's B Medium (Schaad 1980) for 5 days $/ 30^{\circ} \mathrm{C}$.

Seeds of pea (Pisum sativum), Entesar cultivar were obtained from Horticulture Research Institute (HRI), Agriculture Research Center (ARC). Clean seeds with uniform size were used.

Rice straw was collected from unit of Experimental and Agricultural Research, Faculty of Agriculture, Ain Shams University. Chopped rice straw was soaked overnight then sterilized at $121^{\circ} \mathrm{C} / 1 \mathrm{~h}$. to be used as a bed media according to Mohammadi and Abdallah (2007).

The experiment was carried out in $(40 \times 24 \times$ $11 \mathrm{~cm}$ ) trays. $250 \mathrm{~g}$ of sterilized rice straw was added to each tray. Seeding density (dry seeds $/ \mathrm{m}^{2}$ ) was used to produce pea sprout according to (Anwar Dina 2015). Each treatment was repeated three times.

Three semi-automated growth chambers were previously designed for three carbon dioxide concentration treatments (ambient air, 600 and 800 ppm $\mathrm{CO}_{2}$ ) using carbon dioxide pumping as reported by Yossife et al (2017). 


\section{Green pea sprout response to microbial inoculation and increasing atmospheric 2515 $\mathrm{CO}_{2}$ concentration}

\section{Experimental design and treatments}

The experiment was designed in two factorials in complete randomized design with three replicates for each treatment. Factor $\mathrm{A}$ was $\mathrm{CO}_{2}$ concentrations which were three carbon dioxide concentrations (ambient air, 600, and $800 \mathrm{ppm} \mathrm{CO}_{2}$ ). Factor $\mathrm{B}$ was the addition of microbial inoculants which were Azotobacter chroococcum, Bacillus megaterium and Pseudomonas fluorescens and different combinations between all of them.

The experiment was subjected to three groups of treatments. Each group were subjected to one of $\mathrm{CO}_{2}$ concentrations and eight sub-treatments from different combinations of microbial inoculants (Control, Azotobacter chroococcum, Bacillus megaterium, Pseudomonas fluorescens, (Az. chroococcum $+B$. megaterium $),(A z$. chroococcum + Ps. fluorescens), (B. megaterium + Ps. fluorescens), (Az. chroococcum $+B$. megaterium $+P s$. fluorescens).

\section{Addition of microbial inoculants}

Bacillus megaterium $10^{8} \mathrm{cfu} / \mathrm{ml}$ was added 10 days before the cultivation of seeds, while Azotobacter chroococcum and Ps. fluorescens $10^{8}-10^{9}$ $\mathrm{cfu} / \mathrm{ml}$ were added during the experiment; the $1^{\text {st }}$ addition was after pea sprouts emergence and the $2^{\text {nd }}$ addition was after the $1^{\text {st }}$ cut.

Green sprouts (14 days old for $1^{\text {st }}$ cut and 12 days old for $2^{\text {nd }}$ cut) were harvested and dried in an oven at $60 \mathrm{C}^{\circ}$ for $72 \mathrm{hr}$. for measuring sprout characteristics and chemical analysis (proximate and minerals determinations).

\section{Growth parameters}

The following parameters were measured in both first and second cut: Shoot length $(\mathrm{cm})$, shoot fresh and dry weight $\left(\mathrm{g} / \mathrm{m}^{2}\right)$, chlorophyll content $(\mu \mathrm{g}$ Chl. /cm) using SPAD.

\section{Chemical and biochemical parameters}

Mineral contents (P\%, K\%, Ca\% and $\mathrm{Mg} \%, \mathrm{Fe}$ ppm and $\mathrm{Zn} \mathrm{ppm),} \mathrm{proximate} \mathrm{analysis} \mathrm{(moister,}$ protein, lipids, carbohydrates, fiber and ash) were measured according to AOAC (2012). The energy value was calculated using the Atwater factor method $[(9 \times$ fat $)+(4 \times$ carbohydrate $)+(4 \times$ protein)] as described by (Nwabueze 2007).
The data were statistically analyzed using the CoStat package program (Version 6.303; CoHort Software, USA) by ANOVA analysis of variance using completely randomized design two ways with replication, and compare the means by Duncan's Multiple Range Test (Waller and Duncan 1969). All statistical determinations were made at $p \leq$ 0.05 .

\section{RESULTS AND DISCUSSION}

Effect of $\mathrm{CO}_{2}$ concentrations, microbial inoculants, and their interactions on green pea shoots length, weight, and chlorophyll

Green pea shoots are simply pea sprout cuts at early stages of growth before stem branches initiation. Data presented in Table (1) show that generally, first cut recorded increase in shoot length, shoot fresh weight and shoot dry weight when compared to the second cut in all treatments. While the second cut recorded increase in the chlorophyll reading ( $\mu \mathrm{g} \mathrm{Chl} / \mathrm{cm}$ tissue) when compared to the first cut in all treatments. Also, increasing the concentration of $\mathrm{CO}_{2}$ recorded significant increase in all parameters measured at which $800 \mathrm{ppm} \mathrm{CO}$ recorded the highest significant values when compared to $600 \mathrm{ppm}$ and ambient air $\mathrm{CO}_{2}$ in all of shoot length, shoot fresh weight, shoot dry weight and chlorophyll in the first and second cut being 13.19, $12.15 \mathrm{~cm}, 1020,928.5$ $\mathrm{g} / \mathrm{m}^{2}, 154.1,140.2 \mathrm{~g} / \mathrm{m}_{2}$ and $49.8,51.6 \mu \mathrm{g} \mathrm{Chl} . / \mathrm{cm}$ (SPAD), respectively

Concerning microbial inoculants, data show that combined interaction between Azotobacter chroococcum, Bacillus megaterium, and Pseudomonas fluorescens recorded the highest significant increase in shoot length, shoot fresh weight, shoot dry weight and chlorophyll in the first and second cut being 14.03, $12.92 \mathrm{~cm}, 1500.9,1438.2 \mathrm{~g} / \mathrm{m}^{2}$, 226.5, $217.0 \mathrm{~g} / \mathrm{m}^{2}, 51.4$ and $53.0 \mu \mathrm{g} \mathrm{Chl./ \textrm {cm }}$ (SPAD) in respective order.

Regarding the interaction between $\mathrm{CO}_{2}$ concentrations and microbial inoculants, no significant difference was recorded in shoot length in the first cut, shoot fresh weight in the first and second cut and shoot dry weight in the first and second cut. While, pea sprout treated by $800 \mathrm{ppm} \mathrm{CO}_{2}$ and inoculated by $A z$. chroococcum $+B$. megaterium + $P$ s. fluorescens recorded the highest significant shoot length in the second cut and highest significant chlorophyll in the first and second cut being $13.25 \mathrm{~cm}$ and 57.3 and $58.9 \mu \mathrm{g} \mathrm{Chl}$. $/ \mathrm{cm}$ (SPAD), respectively. 
Table 1. Effect of $\mathrm{CO}_{2}$ concentrations, microbial inoculants, and their interactions on green pea sprouts shoot characters and Chlorophyll (Combined data of two experiments)

\begin{tabular}{|c|c|c|c|c|c|c|c|c|c|}
\hline \multirow{3}{*}{$\mathrm{CO}_{2}$} & \multirow{3}{*}{ Microbial inoculants } & \multicolumn{8}{|c|}{ Pea shoot characters } \\
\hline & & \multicolumn{2}{|c|}{$\begin{array}{l}\text { Shoot length } \\
\text { (cm) }\end{array}$} & \multicolumn{2}{|c|}{$\begin{array}{l}\text { Shoot fresh } \\
\text { weight } \mathrm{g} / \mathrm{m} 2\end{array}$} & \multicolumn{2}{|c|}{$\begin{array}{l}\text { Shoot dry weight } \\
\mathrm{g} / \mathrm{m} 2\end{array}$} & \multicolumn{2}{|c|}{$\begin{array}{l}\text { Chlorophyll } \\
\text { SPAD } \\
(\mu \mathrm{g} \mathrm{Chl/cm)}\end{array}$} \\
\hline & & cut & $2^{\text {nd }}$ cut & $1^{\text {st }}$ cut & $1^{\text {st }}$ cut & $2^{\text {nd }}$ cut & $2^{\text {nd }}$ cut & $1^{\text {st }}$ cut & $2^{\text {nd }}$ cut \\
\hline \multirow{8}{*}{ 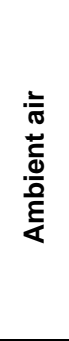 } & & $9 a$ & 10.93 o & $616.4 \mathrm{a}$ & $577.6 \mathrm{a}$ & $89.2 \mathrm{a}$ & $87.1 \mathrm{a}$ & $31.7 \mathrm{n}$ & $5.7 \mathrm{q}$ \\
\hline & Az. chro & $12.52 \mathrm{a}$ & $11.27 \mathrm{~lm}$ & $737.4 \mathrm{a}$ & $692.0 \mathrm{a}$ & $111.2 \mathrm{a}$ & $104.3 \mathrm{a}$ & $37.1 \mathrm{~m}$ & $40.6 \mathrm{n}$ \\
\hline & B. mega & $12.35 \mathrm{a}$ & $11.16 n$ & $652.5 \mathrm{a}$ & $652.6 \mathrm{a}$ & 99.8 a & $98.8 \mathrm{a}$ & $35.5 \mathrm{~m}$ & $36.7 \mathrm{p}$ \\
\hline & Ps. fluol & $12.36 \mathrm{a}$ & $11.20 n$ & $699.4 \mathrm{a}$ & $651.1 \mathrm{a}$ & $105.5 \mathrm{a}$ & $98.2 \mathrm{a}$ & 39.81 & $40.3 n$ \\
\hline & Az. chroococcum $+B$. megaterium & $13.58 \mathrm{a}$ & $12.31 \mathrm{~h}$ & $925.5 \mathrm{a}$ & $863.1 \mathrm{a}$ & $139.5 \mathrm{a}$ & $130.1 \mathrm{a}$ & $41.8 \mathrm{k}$ & $44.4 \mathrm{k}$ \\
\hline & Az. chroococcum $+P$ s. fluorescens & $13.61 \mathrm{a}$ & $12.37 \mathrm{~g}$ & 969.9 a & $935.3 \mathrm{a}$ & $146.2 \mathrm{a}$ & $141.0 \mathrm{a}$ & $44.7 \mathrm{ij}$ & $46.1 \mathrm{i}$ \\
\hline & B. $m$ & $13.56 a$ & $12.29 \mathrm{~h}$ & $808.1 \mathrm{a}$ & 765.5 a & $121.8 \mathrm{a}$ & $115.4 \mathrm{a}$ & $41.5 \mathrm{k}$ & $42.7 \mathrm{~lm}$ \\
\hline & $\begin{array}{r}\text { Az. chroococcl } \\
+P S . f\end{array}$ & $13.91 \mathrm{a}$ & $12.64 \mathrm{e}$ & $1416.8 \mathrm{a}$ & $1379.3 \mathrm{a}$ & $213.7 \mathrm{a}$ & $208.0 \mathrm{a}$ & 47.0gh & $7.2 \mathrm{~h}$ \\
\hline \multicolumn{2}{|r|}{ Mean } & 13. & 7C & $\mathrm{C}$ & $814.6 \mathrm{C}$ & $4 \mathrm{C}$ & 122.9B & C & .... \\
\hline \multirow{8}{*}{ 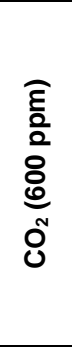 } & Cont & $\mathrm{a}$ & $n$ & 2 & $636.4 \mathrm{a}$ & $105.7 \mathrm{a}$ & & m & 50 \\
\hline & Az. ch & a & 11 & 7 a & $756.6 \mathrm{a}$ & $3 \mathrm{a}$ & & & Olm \\
\hline & & $3 a$ & $11.26 \mathrm{~m}$ & $718.0 \mathrm{a}$ & $674.5 \mathrm{a}$ & $108.3 \mathrm{a}$ & $8 \mathrm{a}$ & $2 m$ & $40.0 n$ \\
\hline & Ps. & a & $11.30 \mathrm{~lm}$ & $777.8 \mathrm{a}$ & $711.9 \mathrm{a}$ & $117.4 \mathrm{a}$ & & & 43.31 \\
\hline & Az. chroococcu & $13.67 \mathrm{a}$ & $12.51 \mathrm{f}$ & $1003.2 \mathrm{a}$ & $923.2 \mathrm{a}$ & $151.2 \mathrm{a}$ & $1 \mathrm{a}$ & 45.7hi & $47.4 \mathrm{~h}$ \\
\hline & Az. chrooc & $13.70 \mathrm{a}$ & & $1043.4 \mathrm{a}$ & $989.5 \mathrm{e}$ & $157.3 \mathrm{a}$ & $149.2 \mathrm{a}$ & $47.4 \mathrm{fg}$ & $51.7 \mathrm{f}$ \\
\hline & B. $m$ & $13.66 a$ & $12.49 \mathrm{f}$ & $892.0 \mathrm{a}$ & 828.0 a & $134.5 \mathrm{a}$ & $124.8 \mathrm{a}$ & $41.3 \mathrm{kl}$ & $44.4 \mathrm{k}$ \\
\hline & $\begin{array}{r}\text { Az. chroococe } \\
+P s .\end{array}$ & $14.02 \mathrm{a}$ & $12.87 \mathrm{~b}$ & $1500.9 \mathrm{a}$ & $1440.5 a$ & $226.5 \mathrm{a}$ & $217.3 \mathrm{a}$ & 49.9de & $52.9 \mathrm{e}$ \\
\hline \multicolumn{2}{|r|}{ Mean } & & & & $70.1 \mathrm{~B}$ & & & & \\
\hline \multirow{8}{*}{ 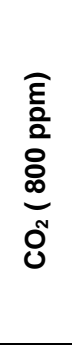 } & & & & $4 \mathrm{a}$ & 695.4 a & $a$ & & $\mathrm{k}$ & $5 \mathrm{~m}$ \\
\hline & $A$ & & & & $815.6 \mathrm{a}$ & & & & \\
\hline & $B$. & $12.52 \mathrm{a}$ & $11.46 \mathrm{jk}$ & $820.6 \mathrm{a}$ & $733.6 \mathrm{a}$ & $124.0 \mathrm{a}$ & $110.9 \mathrm{a}$ & $44.1 \mathrm{j}$ & $45.2 \mathrm{j}$ \\
\hline & Ps. & $12.55 \mathrm{a}$ & $11.50 \mathrm{j}$ & $867.4 \mathrm{a}$ & 770.9 a & $131.1 \mathrm{a}$ & $4 \mathrm{a}$ & 47.1gh & $9.0 \mathrm{~g}$ \\
\hline & Az. chroococcur & $13.75 a$ & $12.70 \mathrm{~cd}$ & $1093.5 a$ & $982.3 \mathrm{a}$ & $165.0 \mathrm{a}$ & $2 \mathrm{a}$ & $53.4 \mathrm{c}$ & $4 \mathrm{c}$ \\
\hline & Az. chroococcum & $13.78 \mathrm{a}$ & $12.73 \mathrm{c}$ & $1137.9 \mathrm{a}$ & $1048.5 \mathrm{a}$ & $171.8 \mathrm{a}$ & $3 \mathrm{~b}$ & $55.4 \mathrm{~b}$ & $56.7 \mathrm{~b}$ \\
\hline & B. megaterium & $13.74 a$ & 12.67de & $972.6 \mathrm{a}$ & $887.0 \mathrm{a}$ & $146.8 \mathrm{a}$ & $133.9 \mathrm{a}$ & $50.4 \mathrm{~d}$ & $53.6 \mathrm{~d}$ \\
\hline & $\begin{array}{r}\text { Az. chroococcum } \\
+ \text { Ps. fluo } \\
\end{array}$ & $\mathrm{a}$ & a & $584.9 \mathrm{a}$ & $1494.8 \mathrm{a}$ & $239.2 \mathrm{a}$ & $225.6 \mathrm{a}$ & $57.3 \mathrm{a}$ & $30.3^{2}+2$ \\
\hline \multicolumn{2}{|r|}{ Mean } & & & $.9 \mathrm{~A}$ & $928.5 \mathrm{~A}$ & & $140.2 \mathrm{~A}$ & & \\
\hline \multirow{8}{*}{ 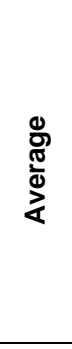 } & Con & $12.28 \mathrm{~F}$ & $3 \mathrm{G}$ & $700.3 \mathrm{H}$ & $636.5 \mathrm{G}$ & $104.5 \mathrm{E}$ & $96.0 \mathrm{E}$ & $4 \mathrm{G}$ & $9 \mathrm{H}$ \\
\hline & Az. chroc & $12.60 \mathrm{D}$ & & $2 \mathrm{E}$ & $754.7 \mathrm{E}$ & 123.7CD & $113.8 \mathrm{CD}$ & E & $0 \mathrm{E}$ \\
\hline & ium & 12.43E & $11.29 \mathrm{~F}$ & $730.4 \mathrm{G}$ & $686.9 \mathrm{~F}$ & 110.7DE & 103.8DE & $6 \mathrm{~F}$ & $40.7 \mathrm{G}$ \\
\hline & Ps. & $12.46 \mathrm{E}$ & $11.33 \mathrm{E}$ & $781.6 \mathrm{~F}$ & $711.3 \mathrm{~F}$ & 118.0DE & 107.3DE & $42.5 \mathrm{E}$ & $44.2 \mathrm{~F}$ \\
\hline & Az. chroococcum $+B$. megaterium & 13.67BC & & $1007.4 \mathrm{C}$ & $922.9 \mathrm{C}$ & & & $46.9 \mathrm{C}$ & $49.0 \mathrm{C}$ \\
\hline & Az. chroococcum $+P$ s. fluorescens & $13.70 \mathrm{~B}$ & $12.55 \mathrm{~B}$ & $1050.4 \mathrm{~B}$ & $991.1 \mathrm{~B}$ & $158.4 \mathrm{~B}$ & $149.5 \mathrm{~B}$ & $49.2 \mathrm{~B}$ & $51.5 \mathrm{~B}$ \\
\hline & B. megaterium + Ps. fluorescens & $13.65 \mathrm{C}$ & $12.48 \mathrm{C}$ & $890.9 \mathrm{D}$ & $826.8 \mathrm{D}$ & $134.4 \mathrm{C}$ & $124.7 \mathrm{C}$ & $44.4 \mathrm{D}$ & $46.9 \mathrm{D}$ \\
\hline & $\begin{array}{c}\text { Az. chroococcum }+ \text { B. megaterium } \\
+ \text { Ps. fluorescens }\end{array}$ & $14.03 \mathrm{~A}$ & $12.92 \mathrm{~A}$ & $1500.9 \mathrm{~A}$ & $1438.2 \mathrm{~A}$ & $226.5 \mathrm{~A}$ & $217.0 \mathrm{~A}$ & $51.4 \mathrm{~A}$ & $53.0 \mathrm{~A}$ \\
\hline \multirow{3}{*}{ نُ } & $\mathrm{CO}_{2}$ concentration & & & 543 & $15 . \angle 400$ & $9.1<50$ & & 177 & 552 \\
\hline & Bio-fer & 0.0294 & 0.0277 & 25.7268 & 24.9012 & 14.9024 & 13.7388 & 0.8453 & 0.4168 \\
\hline & $\mathrm{CO}_{2} \times$ biofertilizer & NS & 0.0481 & NS & NS & NS & NS & 1.4641 & 0.7218 \\
\hline
\end{tabular}

Means in each column in each group followed by the same letter are not significantly different at the $5 \%$ level.

NS= not significant 


\section{Green pea sprout response to microbial inoculation and increasing atmospheric 2517 $\mathrm{CO}_{2}$ concentration}

Proximate analysis and energy of pea sprout cuts as affected by microbial inoculants, $\mathrm{CO}_{2}$ concentrations, and their interactions

The results of the proximate analysis and energy of pea sprout shoot cut $\left(1^{\text {st }}\right.$ cut $)$ are summarized in Table (2). Pea shoot cut showed marked increase in moisture, protein, lipids and ash composition by increasing $\mathrm{CO}_{2}$ concentration at which $800 \mathrm{ppm} \mathrm{CO}_{2}$ recorded the highest significant results when compared to ambient air (control) being $5.40,42.5,4.53$ and $10.39 \%$ in respective order. While carbohydrate, crude fiber, and energy significantly decreased by increasing $\mathrm{CO}_{2}$ concentration at which the highest significant decrease was recorded within 800 ppm $\mathrm{CO}_{2}$ being 28.80, 8.34\% and $326 \mathrm{kcal} / \mathrm{g}$ plant, respectively.

Concerning microbial inoculants, Table (2) show that sprout inoculated by combined interaction between $A z$. chroococcum $+B$. megaterium + $P s$. fluorescens recorded the highest significant increase in moisture, protein, lipids, and ash, as well as the highest significant decrease in carbohydrate and crude fiber, being 5.60, 44.1, 4.55 and $10.28 \%, 27.21,8.26 \%$ respectively. While the highest significant decrease in energy was recorded with sprout inoculated by $A z$. chroococcum only being $325.85 \mathrm{kcal} / \mathrm{g}$.

Regarding the interaction between $\mathrm{CO}_{2}$ concentrations and microbial inoculants, no significant differences were recorded between the treatments in moisture and crude fiber in the sprout. While pea sprout treated by $800 \mathrm{ppm} \mathrm{CO}_{2}$ and inoculated by $A z$. chroococcum+ B. megaterium + Ps. fluorescens recorded the highest significant increase in protein, lipids, and ash as well as the highest significant decrease in carbohydrate being 48.65 , $4.95,10.69$ and $21.77 \%$, respectively. While the highest significant decrease in energy was recorded within sprout treated by $600 \mathrm{ppm} \mathrm{CO}_{2}$ and inoculated by $A z$. chroococcum+ B. megaterium + $P$ s. fluorescens being $324.31 \mathrm{kcal} / \mathrm{g}$.

Minerals content of pea sprout cuts as affected by $\mathrm{CO}_{2}$ concentrations, microbial inoculants, and their interactions

Data in Table (3) generally show that cutting pea sprout shoot 14 days after seed sowing ( $1^{\text {st }}$ cut) recorded increase in all minerals values with medium $\mathrm{CO}_{2}$ concentration ( $600 \mathrm{ppm}$ ) followed by ambient air concentration while the lowest minerals values of $\mathrm{P}, \mathrm{K}, \mathrm{Ca}, \mathrm{Mg}, \mathrm{Fe}$ and $\mathrm{Zn}$ were recorded with higher $\mathrm{CO}_{2}$ concentration (800 ppm). These data indicated that increasing $\mathrm{CO}_{2}$ concentration to $800 \mathrm{ppm}$ affected the translation of minerals from pea seed's cotyledons and roots to pea sprout shoots which recorded the lower contents.

Regarding microbial inoculants, Table (3) shows that inoculating pea sprout by combination of $A z$. chroococcum+ B. megaterium + Ps. fluorescens recorded the highest significant increase in all minerals content in green pea sprout being $0.419,2.391,3.11,0.508 \%, 85.3$ and $51.1 \mathrm{ppm}$, respectively, followed by those inoculated by $A z$. chroococcum+ Ps. fluorescens being $0.360,2.281$, 2.993, $0.469 \%$ and 83.0, $49.1 \mathrm{ppm}$.

The combined interaction between $\mathrm{CO}_{2}$ concentration and microbial inoculants reveal that no significant difference in the $\mathrm{K} \%$ and $\mathrm{Fe} \mathrm{ppm}$ in pea sprout.

The highest significant mineral values of $\mathrm{P}, \mathrm{Ca}$, $\mathrm{Mg}, \mathrm{Fe}$ and $\mathrm{Zn}$ were recorded with pea sprout treated by $600 \mathrm{ppm} \mathrm{CO}_{2}$ and inoculated by combination of $A z$. chroococcum $+B$. megaterium + Ps. fluorescens being $0.545,3.535,0.620 \%$ and $61.3 \mathrm{ppm}$, respectively. Followed by those inoculated by $A z$. chroococcum + Ps. fluorescens in the same concentration being $0.460,3.390,0.588 \%$ and $57.3 \mathrm{ppm}$ respectively. While the lowest $\mathrm{P}, \mathrm{Ca}$, $\mathrm{Mg}$ and $\mathrm{Zn}$ values were recorded inpea sprouts treated by $800 \mathrm{ppm} \mathrm{CO}_{2}$ either un-inoculated by microbial inoculants (control) or inoculated by $B$. megaterium.

Carbone dioxide $\left(\mathrm{CO}_{2}\right)$ level increased in the atmosphere from $270 \mathrm{ppm}$ two hundred years ago to $370-400 \mathrm{ppm}$ today due to fossil fuel use and deforestation, and it is expected to double its concentrations in the coming centuries. Most researchers focus on the growth performance of plants as affected by elevated $\mathrm{CO}_{2}$ due to their ability to acquire $\mathrm{CO}_{2}$ through photosynthesis. Therefore, different researchers reported that elevating $\mathrm{CO}_{2}$ directly improve photosynthetic processes in plants especially those with the $\mathrm{C}_{3}$ photosynthetic pathway suggesting a wide range of physiological, biochemical and morphological responses (Amthor 2001; Kimball et al 2002; Leakey et al 2009 and Yossife et al 2017). In general, higher $\mathrm{CO}_{2}$ concentrations increase plant production due to higher rates of photosynthesis and water utilization (Ludwig and Asseng 2006). Results obtained in this investigation are in line with those reported by Jitla et al (1997); Sage Rowan (2002) and Centritto et al (1999) who mentioned that high $\mathrm{CO}_{2}$ generally stimulate the 
Table 2. Effect of $\mathrm{CO}_{2}$ concentrations, microbial inoculants, and their interactions on proximate analysis $(\mathrm{g} / 100 \mathrm{~g})$ and energy $(\mathrm{kcal} / \mathrm{g})$ of green pea sprouts shoot (Combined data of two experiments)

\begin{tabular}{|c|c|c|c|c|c|c|c|c|}
\hline \multirow[b]{2}{*}{$\mathrm{CO}_{2}$} & \multirow[b]{2}{*}{ Microbial inoculants } & \multicolumn{6}{|c|}{ Proximate analysis $\%$} & \multirow[b]{2}{*}{$\begin{array}{c}\text { Energy } \\
\text { (Kcal. /g) }\end{array}$} \\
\hline & & oisture & Protein & Lipids & $\begin{array}{c}\text { Carbohy } \\
\text { drates }\end{array}$ & Fiber & Ash & \\
\hline \multirow{8}{*}{ 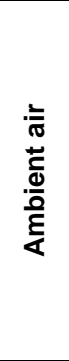 } & اo إ & $4.57 \mathrm{a}$ & $.54 \mathrm{k}$ & $3.35 \mathrm{k}$ & $41.62 \mathrm{a}$ & $8.77 \mathrm{a}$ & 9.161 & $326.75 \mathrm{~cd}$ \\
\hline & Az. chroo & $4.71 \mathrm{a}$ & $36.73 \mathrm{j}$ & $3.55 \mathrm{j}$ & $36.97 \mathrm{~b}$ & $8.62 \mathrm{a}$ & $9.41 \mathrm{k}$ & $326.79 \mathrm{~cd}$ \\
\hline & B. megaterium & $4.63 \mathrm{a}$ & $36.65 \mathrm{j}$ & $3.49 \mathrm{jk}$ & $37.32 \mathrm{~b}$ & $8.55 \mathrm{a}$ & $9.36 \mathrm{k}$ & $327.23 \mathrm{bc}$ \\
\hline & Ps. fluorescens & $4.67 \mathrm{a}$ & $36.66 \mathrm{j}$ & $3.51 \mathrm{jk}$ & $37.23 \mathrm{~b}$ & $8.53 \mathrm{a}$ & $9.39 \mathrm{k}$ & $327.18 \mathrm{bc}$ \\
\hline & $A z$. chroococcum $+B$. megaterium & $4.86 \mathrm{a}$ & $38.25 \mathrm{i}$ & $3.88 \mathrm{i}$ & $35.12 \mathrm{c}$ & $8.47 \mathrm{a}$ & $9.42 \mathrm{k}$ & $328.39 \mathrm{a}$ \\
\hline & Az. chroococcum $+P s$. fluorescens & $4.92 \mathrm{a}$ & $38.33 \mathrm{i}$ & $3.92 \mathrm{i}$ & $34.91 \mathrm{c}$ & $8.44 \mathrm{a}$ & $9.47 \mathrm{jk}$ & $328.27 \mathrm{a}$ \\
\hline & B. megaterium & $4.77 \mathrm{a}$ & $38.22 \mathrm{i}$ & $3.77 \mathrm{i}$ & $35.42 \mathrm{c}$ & $8.43 \mathrm{a}$ & $9.39 \mathrm{k}$ & $328.49 \mathrm{a}$ \\
\hline & $\begin{array}{r}\text { Az. chroococcum } \\
+P s . \text { fluor }\end{array}$ & $5.22 \mathrm{a}$ & $39.21 \mathrm{~h}$ & $4.13 \mathrm{~h}$ & $33.50 \mathrm{e}$ & $8.33 \mathrm{a}$ & $9.61 \mathrm{i}$ & $327.99 a b$ \\
\hline \multicolumn{2}{|r|}{ Mean } & $4.80 \mathrm{~B}$ & $37.0 \mathrm{C}$ & $3.70 \mathrm{C}$ & $36.51 \mathrm{~A}$ & $8.52 \mathrm{~A}$ & $9.40 \mathrm{C}$ & $64 \mathrm{~A}$ \\
\hline \multirow{8}{*}{ 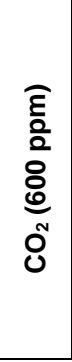 } & & $4.79 \mathrm{a}$ & $39.44 \mathrm{~g}$ & $3.45 \mathrm{jk}$ & $34.06 \mathrm{~d}$ & $8.69 \mathrm{a}$ & $9.56 \mathrm{ij}$ & $9 \mathrm{hi}$ \\
\hline & Az. ch & $2 a$ & $39.87 \mathrm{f}$ & $4.21 \mathrm{gh}$ & $31.98 \mathrm{f}$ & $8.56 a$ & $10.16 \mathrm{gh}$ & $28 \mathrm{fh}$ \\
\hline & & & $39.77 \mathrm{f}$ & $4.11 \mathrm{~h}$ & $32.32 \mathrm{f}$ & $8.49 a$ & $10.11 \mathrm{~h}$ & $38 \mathrm{fh}$ \\
\hline & Ps. flu & $5.23 \mathrm{a}$ & $39.84 \mathrm{f}$ & $4.17 \mathrm{gh}$ & $32.15 \mathrm{f}$ & $8.48 \mathrm{a}$ & 10. & 49fh \\
\hline & $A z$. chroococcum $+B$. megaterium & $5.49 \mathrm{a}$ & $41.56 \mathrm{~d}$ & $4.33 \mathrm{eg}$ & 29.97 ij & $8.45 \mathrm{a}$ & 10. & $325.13 \mathrm{hi}$ \\
\hline & Ps. fluorescens & $5.65 \mathrm{a}$ & $41.59 d$ & $4.44 \mathrm{de}$ & $29.62 j$ & $8.42 \mathrm{a}$ & $10.27 \mathrm{eg}$ & 36 hi \\
\hline & B. megaterium & $5.44 a$ & $41.51 \mathrm{~d}$ & $4.25 \mathrm{fh}$ & $30.23 \mathrm{hi}$ & $8.40 a$ & $10.17 \mathrm{gh}$ & $325.21 \mathrm{gi}$ \\
\hline & $\begin{array}{r}\text { Az. chroococcum } \\
+P s . \text { fluor }\end{array}$ & $5.77 \mathrm{a}$ & $44.45 b$ & $4.56 \mathrm{~cd}$ & 26.36 । & $8.31 \mathrm{a}$ & $10.55 \mathrm{~b}$ & $324.31 \mathrm{i}$ \\
\hline \multicolumn{2}{|r|}{ Mean } & $5 \mathrm{~A}$ & OB & 4.19B & $30.84 \mathrm{~B}$ & $8.47 \mathrm{~A}$ & & $9 \mathrm{C}$ \\
\hline \multirow{8}{*}{ 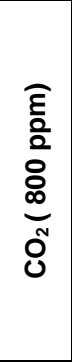 } & Con & $3 \mathrm{a}$ & $39.84 \mathrm{f}$ & $4.16 \mathrm{gh}$ & $32.34 \mathrm{f}$ & $8.62 \mathrm{a}$ & 10. & $2 d g$ \\
\hline & Az. ch & $5.27 \mathrm{a}$ & $40.77 \mathrm{e}$ & $4.43 \mathrm{df}$ & $30.62 \mathrm{gh}$ & $8.56 a$ & & $17 \mathrm{fh}$ \\
\hline & B. $n$ & $5.22 \mathrm{a}$ & $40.63 e$ & $4.27 \mathrm{eh}$ & $31.12 \mathrm{~g}$ & $8.47 a$ & $10.28 \mathrm{dg}$ & 49fh \\
\hline & Ps. fluorescens & $5.23 \mathrm{a}$ & $67 e$ & $4.32 \mathrm{eg}$ & $31.03 \mathrm{~g}$ & $3 a$ & & 67eh \\
\hline & gaterium & $3 a$ & & 4.7 & $27.81 \mathrm{k}$ & & 10. & 326 \\
\hline & Az. chroococcum $+P s$. fluorescens & $5.67 \mathrm{a}$ & $43.33 c$ & $4.75 \mathrm{~b}$ & $27.63 \mathrm{k}$ & $8.17 \mathrm{a}$ & $10.45 \mathrm{bc}$ & $326.56 \mathrm{ce}$ \\
\hline & B. megaterium + Ps. fluorescens & $5.55 \mathrm{a}$ & $43.20 \mathrm{c}$ & $4.63 \mathrm{bc}$ & $28.08 \mathrm{k}$ & $8.15 \mathrm{a}$ & $10.39 \mathrm{ce}$ & $326.79 \mathrm{~cd}$ \\
\hline & $\begin{array}{r}\text { Az. chroococcum } \\
+ \text { Ps. fluo }\end{array}$ & 5 & $48.65 a$ & $4.95 \mathrm{a}$ & $21.77 \mathrm{~m}$ & $8.13 \mathrm{a}$ & $10.69 \mathrm{a}$ & $326.22 \mathrm{df}$ \\
\hline \multicolumn{2}{|r|}{ Mean } & $10 \mathrm{~A}$ & 42.5A & 4.53A & $28.80 \mathrm{C}$ & $8.34 \mathrm{~B}$ & $10.39 \mathrm{~A}$ & 14B \\
\hline \multirow{8}{*}{ 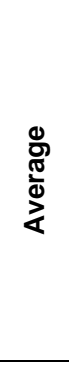 } & & $4.73 \mathrm{E}$ & $37.2 \mathrm{D}$ & $3.65 \mathrm{~F}$ & $36.01 \mathrm{~A}$ & & $9.65 \mathrm{E}$ & $9 \mathrm{C}$ \\
\hline & Az. chr & $7 \mathrm{D}$ & & $4.07 \mathrm{D}$ & $9 \mathrm{C}$ & $8.58 \mathrm{~B}$ & $9.97 \mathrm{CD}$ & $35 \mathrm{C}$ \\
\hline & B. megaterium & $5.01 \mathrm{D}$ & $39.0 \mathrm{C}$ & $3.96 \mathrm{E}$ & $33.59 \mathrm{~B}$ & $8.50 \mathrm{~B}$ & $9.92 \mathrm{D}$ & $326.03 \mathrm{C}$ \\
\hline & Ps. fluorescens & $5.04 \mathrm{D}$ & $39.0 \mathrm{C}$ & 4.00DE & $33.47 \mathrm{BC}$ & $8.48 \mathrm{BC}$ & $9.95 \mathrm{CD}$ & $326.11 \mathrm{BC}$ \\
\hline & $A z$. chroococcum $+B$. megaterium & 5.31BC & & 4.31BC & 30.97DE & 8.37CD & $10.01 \mathrm{BC}$ & $326.77 \mathrm{~A}$ \\
\hline & Az. chroococcum $+P s$. fluorescens & $5.41 \mathrm{~B}$ & $41.0 \mathrm{~B}$ & $4.37 \mathrm{~B}$ & $30.72 \mathrm{E}$ & $8.34 \mathrm{D}$ & 10.06 B & $326.56 \mathrm{AB}$ \\
\hline & B. megaterium + Ps. fluorescens & $5.25 \mathrm{C}$ & $40.9 B$ & $4.22 \mathrm{C}$ & $31.24 \mathrm{D}$ & $8.33 \mathrm{D}$ & $9.98 \mathrm{CD}$ & $326.83 \mathrm{~A}$ \\
\hline & $\begin{array}{c}\text { Az. chroococcum }+B . \text { megaterium } \\
+P s . \text { fluorescens }\end{array}$ & $5.60 \mathrm{~A}$ & $44.1 \mathrm{~A}$ & $4.55 \mathrm{~A}$ & $27.21 \mathrm{~F}$ & $8.26 \mathrm{D}$ & $10.28 \mathrm{~A}$ & 326.17BC \\
\hline \multirow{3}{*}{ نٌ } & $\mathrm{CO}_{2}$ concentration & & & & & & & 0.2934 \\
\hline & Bio-fertilizers & 0.1391 & 0.1305 & 0.0963 & 0.3119 & 0.1105 & 0.0663 & 0.4792 \\
\hline & $\mathrm{CO}_{2} \times$ biofertilizer & NS & 0.2260 & 0.1667 & 0.5402 & NS & 0.1148 & 0.8299 \\
\hline
\end{tabular}

Means in each column followed by the same letter are not significantly different at the $5 \%$ level.

NS= not significant 


\section{Green pea sprout response to microbial inoculation and increasing atmospheric 2519}

$\mathrm{CO}_{2}$ concentration

Table 3. Effect of $\mathrm{CO}_{2}$ concentrations, microbial inoculants, and their interactions on minerals content of pea sprout shoots (Combined data of two experiments)

\begin{tabular}{|c|c|c|c|c|c|c|c|}
\hline \multirow{2}{*}{$\mathrm{CO}_{2}$} & \multirow{2}{*}{ Microbial inoculants } & \multicolumn{6}{|c|}{ Minerals content } \\
\hline & & $\mathbf{P} \%$ & $\mathrm{~K} \%$ & $\mathrm{Ca} \%$ & $\mathrm{Mg} \%$ & $\mathrm{Fe}(\mathrm{ppm})$ & $\mathrm{Zn}(\mathrm{ppm})$ \\
\hline \multirow{8}{*}{ 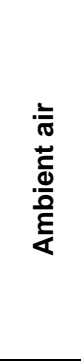 } & Control & $0.232 \mathrm{jk}$ & $1.818 \mathrm{a}$ & $2.780 \mathrm{i}$ & $0.313 \mathrm{k}$ & $67.8 \mathrm{a}$ & $31.3 n$ \\
\hline & Az. chroococcum & $0.288 \mathrm{~h}$ & $1.922 \mathrm{a}$ & $2.885 \mathrm{fg}$ & $0.398 \mathrm{~h}$ & $76.3 \mathrm{a}$ & $41.7 \mathrm{gi}$ \\
\hline & B. megaterium & $0.265 \mathrm{hi}$ & $1.818 \mathrm{a}$ & 2.808 hi & $0.320 \mathrm{k}$ & $71.2 \mathrm{a}$ & $33.0 \mathrm{ln}$ \\
\hline & Ps. fluorescens & $0.328 \mathrm{fg}$ & $1.860 \mathrm{a}$ & $2.840 \mathrm{gh}$ & $0.353 \mathrm{j}$ & $72.0 \mathrm{a}$ & $35.3 \mathrm{kl}$ \\
\hline & $A z$. chroococcum $+B$. megaterium & $0.315 \mathrm{~g}$ & $1.947 \mathrm{a}$ & 2.933 ef & $0.415 \mathrm{gh}$ & $82.2 \mathrm{a}$ & 44.8 ef \\
\hline & Az. chroococcum $+P s$. fluorescens & $0.372 \mathrm{de}$ & $1.985 \mathrm{a}$ & $2.967 \mathrm{e}$ & $0.442 \mathrm{f}$ & $82.2 \mathrm{a}$ & $47.0 \mathrm{de}$ \\
\hline & B. megaterium + Ps. fluorescens & 0.345 ef & $1.885 \mathrm{a}$ & $2.867 \mathrm{~g}$ & $0.365 \mathrm{ij}$ & $76.5 \mathrm{a}$ & $40.7 \mathrm{hi}$ \\
\hline & $\begin{array}{c}\text { Az. chroococcum }+B \text {. megaterium } \\
+ \text { Ps. fluorescens }\end{array}$ & $0.395 d$ & $2.067 \mathrm{a}$ & $3.145 \mathrm{~cd}$ & $0.470 \mathrm{e}$ & $84.0 \mathrm{a}$ & $47.8 d$ \\
\hline \multicolumn{2}{|r|}{ Mean } & $0.318 \mathrm{~B}$ & $1.913 \mathrm{~B}$ & $2.903 \mathrm{~B}$ & $0.385 \mathrm{~B}$ & 76.5B & $40.2 \mathrm{~B}$ \\
\hline \multirow{8}{*}{ 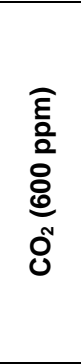 } & Control & $0.320 \mathrm{fg}$ & $2.795 \mathrm{a}$ & $2.868 \mathrm{~g}$ & $0.423 \mathrm{fg}$ & $83.3 \mathrm{a}$ & $43.5 \mathrm{fg}$ \\
\hline & Az. chroococcum & $0.372 \mathrm{de}$ & $2.900 \mathrm{a}$ & $3.091 \mathrm{~d}$ & $0.523 d$ & $89.5 \mathrm{a}$ & $55.0 \mathrm{bc}$ \\
\hline & B. megaterium & $0.342 \mathrm{fg}$ & $2.818 \mathrm{a}$ & 2.930 ef & $0.442 \mathrm{f}$ & $85.7 \mathrm{a}$ & 45.0 ef \\
\hline & Ps. fluorescens & $0.430 \mathrm{c}$ & $2.872 \mathrm{a}$ & $2.965 \mathrm{e}$ & 0.478 e & $87.5 \mathrm{a}$ & $47.3 \mathrm{de}$ \\
\hline & $A z$. chroococcum $+B$. megaterium & $0.375 d$ & $2.922 \mathrm{a}$ & $3.180 \mathrm{c}$ & $0.560 \mathrm{c}$ & $93.0 \mathrm{a}$ & $53.8 \mathrm{c}$ \\
\hline & Az. chroococcum +Ps. fluorescens & $0.460 \mathrm{~b}$ & $2.953 \mathrm{a}$ & $3.390 \mathrm{~b}$ & $0.588 \mathrm{~b}$ & $95.0 \mathrm{a}$ & $57.3 \mathrm{~b}$ \\
\hline & B. megaterium + Ps. fluorescens & $0.442 \mathrm{bc}$ & $2.910 \mathrm{a}$ & $2.960 \mathrm{e}$ & $0.515 d$ & $90.7 \mathrm{a}$ & $48.3 d$ \\
\hline & $\begin{array}{c}\text { Az. chroococcum }+B \text {. megaterium } \\
+P s \text {. fluorescens }\end{array}$ & $0.545 \mathrm{a}$ & $3.067 \mathrm{a}$ & $3.535 \mathrm{a}$ & $0.620 \mathrm{a}$ & $97.2 \mathrm{a}$ & $61.3 \mathrm{a}$ \\
\hline \multicolumn{2}{|r|}{ Mean } & $0.411 \mathrm{~A}$ & $2.905 \mathrm{~A}$ & $3.115 \mathrm{~A}$ & $0.519 \mathrm{~A}$ & $90.2 A$ & $51.5 \mathrm{~A}$ \\
\hline \multirow{8}{*}{ 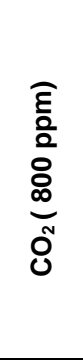 } & Control & $0.158 \mid$ & $1.750 \mathrm{a}$ & $2.470 \mathrm{~m}$ & $0.237 n$ & $59.5 \mathrm{a}$ & $31.2 n$ \\
\hline & Az. chroococcum & $0.208 \mathrm{k}$ & $1.873 \mathrm{a}$ & 2.5561 & $0.325 \mathrm{k}$ & $67.8 \mathrm{a}$ & $36.7 \mathrm{jk}$ \\
\hline & B. megaterium & $0.178 \mid$ & $1.792 \mathrm{a}$ & $2.453 \mathrm{~m}$ & $0.253 \mathrm{mn}$ & $62.3 \mathrm{a}$ & $32.0 \mathrm{mn}$ \\
\hline & Ps. fluorescens & $0.232 \mathrm{jk}$ & $1.803 \mathrm{a}$ & $2.463 \mathrm{~m}$ & 0.2821 & $64.8 \mathrm{a}$ & $33.0 \mathrm{ln}$ \\
\hline & $A z$. chroococcum $+B$. megaterium & $0.208 \mathrm{k}$ & $1.893 \mathrm{a}$ & $2.573 \mathrm{kl}$ & $0.362 \mathrm{ij}$ & $71.7 \mathrm{a}$ & $39.0 \mathrm{ij}$ \\
\hline & Az. chroococcum +Ps. fluorescens & $0.248 \mathrm{ij}$ & $1.905 \mathrm{a}$ & $2.623 \mathrm{jk}$ & $0.377 \mathrm{i}$ & $71.8 \mathrm{a}$ & $43.0 \mathrm{fh}$ \\
\hline & B. megaterium + Ps. fluorescens & $0.262 \mathrm{hi}$ & $1.845 \mathrm{a}$ & $2.537 \mid$ & $0.268 \mathrm{Im}$ & $66.7 \mathrm{a}$ & $34.5 \mathrm{~km}$ \\
\hline & $\begin{array}{c}\text { Az. chroococcum }+B \text {. megaterium } \\
+ \text { Ps. fluorescens }\end{array}$ & $0.318 \mathrm{fg}$ & $2.038 \mathrm{a}$ & $2.673 \mathrm{j}$ & $0.433 \mathrm{fg}$ & $74.8 \mathrm{a}$ & $44.2 \mathrm{fg}$ \\
\hline \multicolumn{2}{|r|}{ Mean } & $0.227 \mathrm{C}$ & $1.863 \mathrm{C}$ & $2.544 \mathrm{C}$ & $0.317 \mathrm{C}$ & $67.4 \mathrm{C}$ & $36.7 \mathrm{C}$ \\
\hline \multirow{8}{*}{ 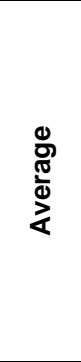 } & Control & $0.237 \mathrm{~F}$ & $2.121 \mathrm{~F}$ & $2.706 \mathrm{G}$ & $0.324 \mathrm{G}$ & $70.2 \mathrm{~F}$ & $35.3 \mathrm{~F}$ \\
\hline & Az. chroococcum & $0.289 \mathrm{D}$ & $2.232 \mathrm{C}$ & $2.844 \mathrm{D}$ & $0.416 \mathrm{D}$ & $77.9 \mathrm{C}$ & $44.4 \mathrm{C}$ \\
\hline & B. megaterium & $0.262 \mathrm{E}$ & 2.143EF & $2.731 \mathrm{FG}$ & $0.338 \mathrm{~F}$ & $73.1 \mathrm{E}$ & $36.7 \mathrm{~F}$ \\
\hline & Ps. fluorescens & $0.330 \mathrm{C}$ & 2.178DE & $2.756 \mathrm{~F}$ & $0.371 \mathrm{E}$ & $74.8 \mathrm{D}$ & $38.6 \mathrm{E}$ \\
\hline & $A z$. chroococcum $+B$. megaterium & $0.299 \mathrm{D}$ & $2.254 \mathrm{BC}$ & $2.896 \mathrm{C}$ & $0.446 \mathrm{C}$ & $82.3 \mathrm{~B}$ & $45.9 \mathrm{C}$ \\
\hline & Az. chroococcum $+P s$. fluorescens & $0.360 \mathrm{~B}$ & $2.281 \mathrm{~B}$ & $2.993 \mathrm{~B}$ & $0.469 \mathrm{~B}$ & $83.0 \mathrm{~B}$ & $49.1 \mathrm{~B}$ \\
\hline & B. megaterium + Ps. fluorescens & $0.349 \mathrm{~B}$ & $2.213 C D$ & $2.788 \mathrm{E}$ & $0.383 \mathrm{E}$ & $77.9 \mathrm{C}$ & $41.2 \mathrm{D}$ \\
\hline & $\begin{array}{c}\text { Az. chroococcum }+B \text {. megaterium } \\
+ \text { Ps. fluorescens }\end{array}$ & $0.419 \mathrm{~A}$ & $2.391 \mathrm{~A}$ & $3.118 \mathrm{~A}$ & $0.508 \mathrm{~A}$ & $85.3 \mathrm{~A}$ & $51.1 \mathrm{~A}$ \\
\hline \multirow{3}{*}{ L.S.D } & $\mathrm{CO}_{2}$ concentration & 0.0090 & 0.0239 & 0.0190 & 0.0074 & 0.7709 & 0.9094 \\
\hline & Bio-fertilizers & 0.0148 & 0.0390 & 0.0310 & 0.0120 & 1.2588 & 1.4850 \\
\hline & $\mathrm{CO}_{2} \times$ biofertilizer & 0.0256 & NS & 0.0537 & 0.0208 & NS & 2.5722 \\
\hline
\end{tabular}

Means in each column followed by the same letter are not significantly different at the $5 \%$ level.

$\mathrm{NS}=$ not significant 
photosynthetic process that leads to significant high growth rate. As well, Drake et al (1999) and Amthor (2000) reported that increasing atmospheric $\mathrm{CO}_{2}$ is causing respiratory inhibition and water balance of the plants that stimulates plant growth and yield. Also, Stephen et al (2011) reported that elevated $\mathrm{CO}_{2}$ stimulates photosynthesis that leads to increase carbon (C) uptake and assimilation, thereby increasing plant growth.

The important of PGPB could be manifested on their ability to excrete phytohormones such as auxins and gibberellins, etc., thereby improving the growth and early development of plants (Zayed Mona 2012; Ba'konyi et al 2013; Zayed Mona et al 2013 and Selim and Zayed Mona 2017). In this subject, (Kloepper and Beauchamp 1992) mentioned that inoculation of wheat by the Azotobacter $\mathrm{sp}$. and Bacillus sp. increased its yield. Based on our results, microbial inoculant treatments improved the growth performance of pea when compared to control at which the combination between Azotobacter chroococcum, Bacillus megaterium and Pseudomonas fluorescens recorded the best effect on plant growth performance which could be displayed in shoot length, fresh and dry weight, which agree with the results mentioned by Zayed Mona (2012) and and Selim and Zayed Mona (2017). Also, Cakmakci et al (2007) reported that inoculation of plants with $\mathrm{N}_{2}$ fixing bacteria significantly increased the uptake of $\mathrm{N}, \mathrm{Fe}, \mathrm{Mn}$, and $\mathrm{Zn}$ by barley seedlings when compared to the uninoculated plants (control). Also, plant responses to $N_{2}$ fixing bacteria could be associated with other mechanisms, rather than direct $\mathrm{N}_{2}$ fixation such as production of hormones which has been suggested as one of the mechanisms by which PGPR stimulate plant growth (Cakmakci et al 2007).

\section{REFERENCES}

Abd El- Malek Y. and Ishac Y.Z. 1968. Evaluation of methods used in counting azotobacter. J. Appl. Microbiol. 31(3), 267-275.

Abdallah M.M.F. 2008.Seed sprouts, a pharaoh's heritage to improve food quality. Arab Univ. J. Agric. Sci. 16(2), 469-478.

Ahemad M. and Khan M.S. 2011. Effects of insecticides on plant-growth-promoting activities of phosphate solubilizing rhizobacterium Klebsiella sp. strain PS19. Pesticide Biochem. Physiol. 100(1), 51-56.
Ahmed E.A.A.S., Abo El-Azam Nashwa A.L., ElBehairy U.A.A. and Abdallah M.M.F. 2018. Organic pea sprout indoor to improve pie quality. Arab Univ., J. Agric., Sci., Ain Shams Univ., Cairo, Special ISSUE, 26(2), (in press).

Amthor J.S. 2000. The McCree-de-Wit-Penningde-Vries-Thornley respiration paradigms: 30 years later. Ann. Bot. 86, 1-20.

Amthor J.S. 2001. Effects of atmospheric $\mathrm{CO}_{2}$ concentration on wheat yield: a review of results from experiments using various approaches to control $\mathrm{CO}_{2}$ concentration. Field Crops Res.73, 1-34.

Anwar Dina A. 2015. Effect of number of harvests on the production and nutritive value of mushroom fruits, sprout vegetables and their residues. Ph.D. Thesis, in Horticulture (vegetable crops) Fac. Agric., Ain Shams Univ., Cairo, Egypt, $139 \mathrm{p}$.

AOAC 2012. Official Methods of Analysis of AOAC International. $\left(19^{\text {th }}\right.$ ed.) Dumes method. No.968.06. Chapter 4, 25-26.

Atta H.M., Selim S.M. and Zayed, Mona S. 2012. Natamycin antibiotic produced by Streptomyces sp.: Fermentation, purification and biological activities. J. Am. Sci. 8(2), 469-475.

Bákonyi N., Bott S., Gajdos E'., Szab A., Jakab A., Toth B., Makleit P., Veres Sz. 2013. Using biofertilizer to improve seed germination and early development of maize. Pol. J. Environ. Stud. 22(6), 1595-1599.

Cakmakci R., Donmez M.F., Erdogan U. 2007. The effect of plant growth promoting rhizobacteria on barley seedling growth, nutrient uptake, some soil properties, and bacterial counts. Turk. J. Agric. For. 31, 189-199.

Centritto M., Lee H.J. and Jarvis P.G. 1999. Increased growth in elevated $\left[\mathrm{CO}_{2}\right]$ : An early, short-term response? Glob. Change Biol. 5, 623-633.

Drake B.G., Azcon-Bieto J., Berry J., Bunce J., Dijkstra P., Farrar J., Gifford R.M., GonzalezMeler M.A., Koch G., Lambers H., Siedow J. and Wullschleger S. 1999. Does elevated atmospheric $\mathrm{CO}_{2}$ concentration inhibit mitochondrial respiration in green plants? Plant Cell Environ. 22, 649-657.

Gray W.M. 2004. Hormonal regulation of plant growth and development. PLoS Biol. 2(9), 1270-1273. 
Ibrahim Mona I. 2015. Postharvest physiology of some vegetable crops. Ph.D. Thesis, in Horticulture (vegetable crops), Fac. Agric., Ain Shams Univ., Cairo, Egypt. , 165 p.

IPCC, 2001. Climate Change. The Scientific Basis . Houghton J.T.; Ding Y.; Griggs D.J.; Noguer M.; van der Linden P.J.; Dai X.; Maskell K. and Johnson C.A. eds, 881. Cambridge University Press, Cambridge, UK.

Jacobs M.B. and Gerstein M.J. 1960. Handbook of Microbiology. Van D. (Ed) Nostrand Co., pp. 139-202. Inc., New York, USA.

Jitla D.S., Rogers G.S., Seneweera S.P., Basra A.S., Oldfield R.J. and Conroy J.P. 1997. Accelerated early growth of rice at elevated CO2. Plant Physiol. 115, 15-22.

Kimball B.A., Kobayashi K. and Bindi M. 2002. Responses of agricultural crops to free-air $\mathrm{CO}_{2}$ enrichment. Adv. Agron. 77, 293-368.

Kloepper J.W. and Beauchamp C.J. 1992. A review of issues related to measuring of plant roots by bacteria. Can. J. Microbiol. 38(12), 1219-1232.

Leakey A.D.B., Ainsworth E.A., Bernacchi C.J., Rogers A., Long S.P. and Ort D.R. 2009. Elevated $\mathrm{CO}_{2}$ effects on plant carbon, nitrogen, and water relations: six important lessons from FACE. J. Exp. Bot. 60, 2859-2876.

Ludwig F. and Asseng S. 2006. Climate change impacts on wheat production in a Mediterranean environment in Western Australia. Agric. Sys. 90, 159-179.

Mártinez -Sánchez A., Luna M.C., Selma M.V., Tudela J.A., Abad J. and Gill M.I. 2012. Babyleaf and multi-leaf of green and red lettuces are suitable raw materials for the fresh-cut industry. Postharvest Biol. Technol. 63(1), 1-10.

Márton M., Mándoki Z., Csapo'Kiss Z. and Csapo' J. 2010. The role of sprouts in human nutrition. A review. Acta Univ. Sapientiae 3, 81-117.

Mcdaniel M.D., Tiemann L.K. and Grandy A.S. 2014. Does agricultural crop diversity enhance soil microbial biomass and organic matter dynamics? A meta-analysis. Ecol. Appl. 24(3), 560-570.

Miles C.A. and Sonde M. 2003. Pea shoots. Pacific Northwest Extension Publications, PNW 567, 1-8.

Mohammadi Thanaa F. and Abdallah M.M.F. 2007. Effect of four seed sprouts on rice straw and spent mushroom media of rice straw to be used as a green fodder. Egyptian. J. Nutrition Feeds 10(2), 679-691.
Nwabueze T.U. 2007. Nitrogen solubility index and amino acid profile of extruded African breadfruit (T. Afiricano) blends. Nig. Food. J. 25, 35-35.

Ortíz-Castro R., Valencia-Cantero E. and López-Bucio J. 2008. Plant growth promotion by Bacillus megaterium involves cytokinin signaling. Plant Signaling \& Behavior 3(4), 263265.

Penas E., Gomez R., Frias J. and Vidal-Valverde C. 2008. Application of high - pressure on alfalfa (Medigo sativa) and mung bean (Vigna radiate) seeds to enhance the microbiological safety of their sprouts. Food Control 19, 698-705.

Poudel M. and Dunn B. 2017. Greenhouse carbon dioxide supplementation. Oklahoma cooperative extension service march sheet. HLA67236 p. (http://osufacts.okstate.edu).

Rico D., Martin-Diana A.B., Barat J.M. and Barry-Ryan C. 2007. Extending and measuring the quality of fresh-cut fruit and vegetable. A review: Trends Food Sci. Technol. 18(7), 373386.

Sage Rowan F. 2002. How terrestrial organisms sense, signal, and respond to carbon dioxide. Integ. Comp. Biol. 42, 469-480.

Santos J., Herrero M., Mendiola J.A., OlivaTeles M.T., Ibáfiez E., Delerue-Matos C. and Oliveira M.B.P.P. 2014. Assessment of nutritional and metabolic profiles of pea shoot: the new ready-to-eat baby-leaf vegetable. Food Res. Int. 58, 105-111.

Schaad N.W. 1980. Laboratory Guide for the Identification of Plant Pathogenic Bacteria. Schaad N.W.; Jones J.B.; and Chun W. (eds.), 72 p. The American Phytopathological Society, St. Paul, MN, USA.

Selim Sh.M. and Zayed Mona S. 2017. Microbial Interactions and Plant Growth. In: PlantMicrobe Interactions in Agro-Ecological Perspectives. Volume 1: Fundamental Mechanisms, Methods and Functions pp. 1-15. Springer, India.

Stephen A.P., Runion G.B., Marble S.C., Rogers H.H., Gilliam C.H. and Torbert Allen H. 2011. A review of elevated atmospheric $\mathrm{CO}_{2}$ effects on plant growth and water relations: implications for horticulture. Hortscience, 46(2), 158162.

Süß A., Danner M., Obster C., Locherer M., Hank T. and Richter K. 2015. Measuring Leaf Chlorophyll Content with the Konica Minolta SPAD-502Plus - Theory, Measurement, Problems, Interpretation. EnMAP Field Guides 
Technical Report, GFZ Data Services. pp. 113.

Valentine A.J., Benedito V.A. and Kang Y. 2010. Legume nitrogen fixation and soil abiotic stress: from physiology to genomics and beyond. In: Nitrogen metabolism in plants in the postgenomic era, Foyer, C/ Zhao M. (eds). pp. 207-248, Wiley-Blackwell, Oxford.

Van Loon L.C. 2007. Plant responses to plant growth-promoting rhizobacteria. Eur. J. Plant Pathol. 119, 243-254.

Waller R.A. and Duncan D.B. 1969. A Bayes rule for the symmetric multiple comparison problems. J. Amer. Stat. Assoc. 64, 1484-1503.
Yossife H., Eldanasoury M.M., Fayd T.B., Hassanein M.K. and Ali B.A.A. 2017. Impact of climate change conditions on some chemical compounds of wheat as indicators for photosynthetic efficiency. NY. Sci. J. 10(9), 104-110.

Zayed Mona S. 2012. Improvement of growth and nutritional quality of Moringa oleifera using different biofertilizers. Ann. Agric. Sci. 57, 5362.

Zayed Mona S., Hassanein M.K.K., Eissa Nahed H. and Abdallah M.M.F. 2013. Productivity of pepper crop (Capsicum annuum L.) as affected by organic fertilizer, soil solarization and endomycorrhizae. Ann. Agric. Sci. 58(2), 131-137. 
مجلة اتحاد الجامعات العربية للعلوم الزراعية ، جامعة عين شمس ، القاهرة

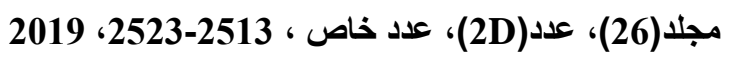

Website: http://strategy-plan.asu.edu.eg/AUJASCl/

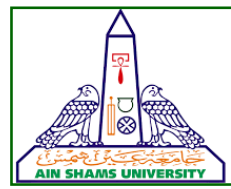

2523

\section{استجابة نبت البسلة الخضراء للأضافات الميكروييه وزيادة تركيز ثاني أكسيد الكريون في الغلاف الجوي}

[185]

ناهد حسن عيسى" 1 - منى سعيد زايد² - مسعد قطب حسنين 1 - ممدوح محمد فوزى عبدالله 3

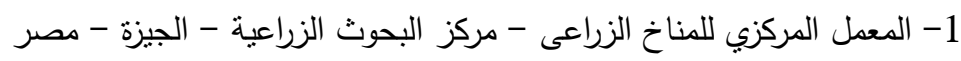

2- قسم الميكروبيولوجيا الزراعيه - كلية الزراعة - جامعة عين شمس - صندوق بريد 68 - حدايق شبرا 11241 -

3- قسم البساتين- كلية الزراعة - جامعة عين شمس - صندوق بريد 68 - حدايق شبرا 11241 - القاهرة - مصر

*Corresponding author: monaszayed@agr.asu.edu.eg

Received 9 June, 2018

Accepted 4 July, 2018

المليون من ثاني أكسيد الكربون مع زيادة حوالي 20٪

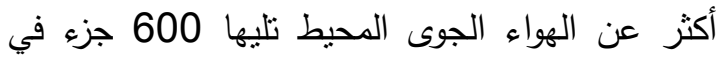
المليون مع زيادة حوالي 9.4٪ من ثناني أكسيد الكربون كان $\mathrm{CO}_{2}$ كانت هناك زيادة فى محتوى البروتين الخام الكلى من فن ون

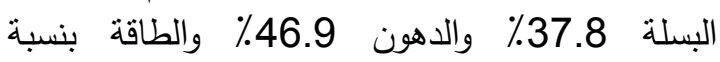

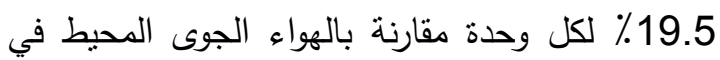

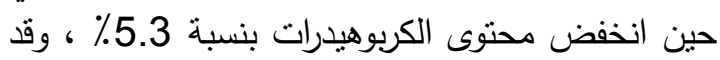

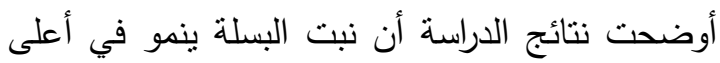
تركيز من ثاني أكسيد الكربون مع الحفاظ على الجنى الجودة

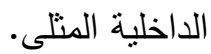

الكلمات الدالة: البسلة، النبت،

$$
\text { الميكروبيه }
$$

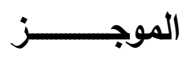

يناثر انتاج نبت البسلة الاخضر Pisum sativum

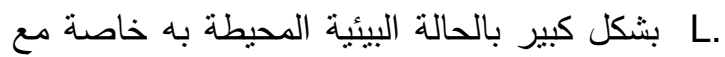

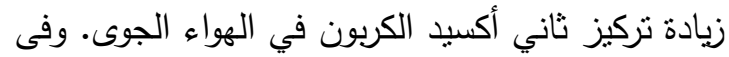

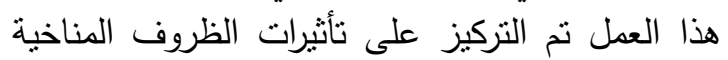

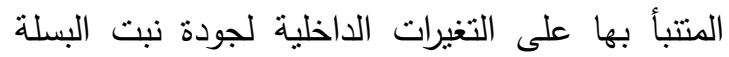
باستخدام نركيز ثاني أكسيد الكربون بمقدار 600

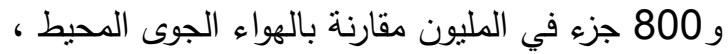

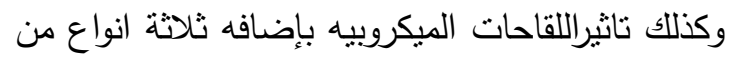

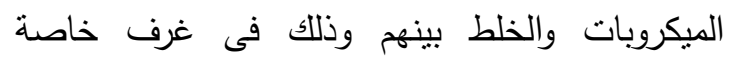
للإنبات نصف اتوماتيك.

وقد أظهرت النتائج أن أكبر إنتاجية من نبت البسلة

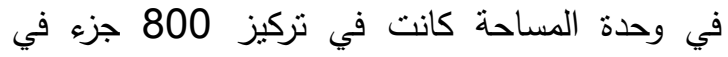


